\title{
Rebrota de Lotus spp. de diferentes hábitos de crescimento ${ }^{1}$
}

\section{Rita Poles Maroso², Simone Meredith Scheffer-Basso ${ }^{3}$, Cercí Maria Carneiro $^{3}$}

\author{
${ }^{1}$ Parte da dissertação de Mestrado da primeira autora. Curso de Pós-Graduação em Agronomia, Universidade de Passo Fundo, RS. \\ 2 Bióloga, MSc. \\ 3 Universidade de Passo Fundo, RS. Campus I, Bairro São José, Passo Fundo, CEP: 99001970.
}

RESUMO - Este trabalho foi realizado com o objetivo de comparar cultivares de Lotus spp. de diferentes hábitos de crescimento (L. corniculatus: São Gabriel - semi-ereto; ARS2620 - prostrado; L. uliginosus: cv. Maku - decumbente) cultivados em vasos e submetidos a diferentes alturas $(4-8 \mathrm{~cm}$ ) e freqüências (quinzenal - mensal) de corte do 95으 ao 233으 dia de idade. As maiores produções de massa seca (g/planta) foram obtidas com cortes mensais a $8 \mathrm{~cm}$ (Maku = 13,3; S. Gabriel = 11,5; ARS2620 = 7,3). Em níveis intermediários (mensal - $4 \mathrm{~cm}$; quinzenal - $8 \mathrm{~cm}$ ), o cultivar Maku foi superior aos demais, enquanto, em cortes quinzenais $(4 \mathrm{~cm}$ ), não houve diferença entre os cultivares. O cultivar ARS2620 apresentou menor estatura e maior cobertura verde residual após os cortes. Na condição residual após o último corte, o cultivar Maku superou os demais quanto ao diâmetro da coroa e às produções de MS da raiz primária, subterrânea, aérea e de sóboles. Todos os cultivares foram favorecidos pelo manejo menos intenso, mas apresentaram mecanismos compensatórios ao serem manejados com cortes baixos em menor freqüência ou com cortes freqüentes, mas menos intensos. Os três cultivares apresentaram caules subterrâneos sobolíferos. A sobrevivência do cultivar São Gabriel e o sistema subterrâneo de Lotus spp. foram comprometidos em cortes intensos e freqüentes.

Palavras-chave: cornichão, manejo, produção, sobrevivência

\section{Regrowth of Lotus spp. with different growth habits}

\begin{abstract}
This work aimed to compare Lotus spp. cultivars with different growth habits (L. corniculatus: cv. São Gabriel, semi-erect, cv. ARS2620, prostrate, and L. uliginosus: cv. Maku, decumbent), submitted to different height (4 - $8 \mathrm{~cm})$ and cutting frequencies $\left(15 \mathrm{~d}\right.$ and $30 \mathrm{~d}$ ), between the $95^{\text {th }}$ and $233^{\text {rd }}$ days old, grown in pot. Greater DM production (g. plant ${ }^{-1}$ of DM) was obtained under monthly cutting at $8 \mathrm{~cm}($ Maku = 13.3; S. Gabriel = 11.5; ARS2620 = 7.3). Maku surpassed the others when intermediate levels (monthly cutting-4 cm; fortnighly cutting- $8 \mathrm{~cm}$ ) were used; harvests every $15 \mathrm{~d}$ and at $4 \mathrm{~cm}$ resulted in no difference among cultivars. The cv. ARS2620 showed the lowest height and the highest residual green canopy after defoliation. After the last cut, the cv. Maku overcame the other cultivars regarding crown diameter, DM of taproot, DM of soboles, DM of underground roots, and shoots. Lenient management favoured all the cultivars but they showed compensatory mechanism when they were submitted to intense and less frequent cutting or under frequent and less intense cutting. The three cultivars showed subterraneum stems of sobole type. The persistence of cv. S. Gabriel and the underground system of Lotus spp. were affected under intense and frequent cutting.
\end{abstract}

Key Words: birdsfoot trefoil, management, production, survival

\section{Introdução}

Uma das propriedades fundamentais das plantas forrageiras perenes é sua capacidade de reiniciar novo crescimento após desfolhações. A rapidez como reiniciam o crescimento após corte ou pastejo é o principal fator que determina sua produtividade no próximo corte e sua produção na estação de crescimento. Esse processo depende das condições ambientais, como temperatura, umidade e fertilidade do solo, assim como de suas condições morfofisiológicas por ocasião do corte. Entre os caracteres morfofisiológicos que condicionam o potencial da rebrota e influenciam a taxa de crescimento das forrageiras, destacam-se o nível de carboidratos de reserva da planta, a altura dos pontos de crescimento em relação ao plano de corte, a área foliar remanescente e o número e a viabilidade de gemas basilares (Brougham, 1956; Brown et al., 1966).

Segundo Sheaffer et al. (1992), o hábito de crescimento também pode refletir na capacidade de rebrota e no tempo de vida útil da planta. Em estudo com Trifolium ambiguum M. Bieb., rizomatoso e cornichão (Lotus corniculatus L.), sem rizomas, sob pastejo, esses autores verificaram que o cornichão teve seu estande reduzido, ao contrário do observado na primeira planta. Assim, a existência de caules 
subterrâneos ou estolões é importante em plantas utilizadas sob pastejo.

O cornichão é uma leguminosa com problemas de persistência, especialmente sob pastejo, em virtude de seu hábito de crescimento, apesar da versatilidade quanto à sobrevivência do estande, uma vez que o aumento da população dessas plantas pode ser obtido por ressemeadura natural (Caroso et al., 1981; Flaresso \& Saibro, 1992). No caso do cultivar São Gabriel, de hábito semi-ereto, a seleção de tipos morfológicos mais prostrados, com coroas mais vigorosas e caules subterrâneos seria interessante, uma vez que é um cultivar adaptado ao Sul do Brasil e com todas as vantagens do gênero, como não provocar timpanismo e ser mais tolerante à acidez em relação aos trevos (Trifolium spp.) e à alfafa (Medicago sativa L.).

Caroso et al. (1981), ao compararem progênies de clones do cultivar São Gabriel com cultivares americanos, verificaram diferentes graus de persistência em resposta aos cortes e observaram que os mais eretos persistiram menos. A variabilidade São Gabriel em relação à produção e qualidade da forragem sob cortes também foi observada por Soster et al. (2004b), que notaram diferenças quanto ao comprimento dos entrenós, à estatura, ao diâmetro, à produção de forragem e à composição química. Araújo \& Jacques (1974a), com o mesmo cultivar, verificaram que, quanto mais avançado o estádio de crescimento da planta, maior a produção de caules e folhas e das ramificações axilares superiores. Estudos antigos comprovaram que o cornichão, quando não é cortado, produz apenas um crescimento da coroa e que os crescimentos subseqüentes surgem de gemas axilares localizadas ao longo das hastes (Smith, 1962).

Outra espécie do gênero, Lotus uliginosus Schkuhr (cornichão-dos-banhados), apesar de sobolífera (caules subterrâneos), também tem sua rebrota favorecida sob cortes menos intensos (Sheat, 1980). No entanto, sob cortes mais intensos, porém com aumento do intervalo de corte, apresenta boa rebrota, indicando compensação e possibilidade de recuperação das plantas. O hábito prostrado e a presença sóboles têm indicado esse cultivar como uma leguminosa de fácil adaptação ao pastejo. Apesar disso, segundo Wen et al. (2002), ainda são poucos os estudos sobre a rebrota em genótipos rizomatosos.

A partir de materiais de cornichão classificados como rizomatosos coletados no Marrocos, cruzados com o tipo comum (sem rizomas), Beuselinck \& Steiner (1996) obtiveram cultivares com caules subterrâneos, como o ARS2620. No entanto, Maroso et al. (2004), a partir de estudos sobre a anatomia em cornichão e com base nos critérios de classificação de estruturas subterrâneas divulgadas por Appezatto da Glória (2003), verificaram que os caules subterrâneos do cornichão são sóboles e não rizomas, pois não se originam da plúmula e dão origem a novos caules aéreos, ao contrário dos rizomas típicos.

Este trabalho foi realizado com o objetivo de comparar a capacidade de rebrota e a sobrevivência de três cultivares de Lotus spp. com diferentes hábitos de crescimento manejados em diferentes alturas e intervalos de corte. Observaram-se a resposta e a proporção das respostas (produção, dimensão, sobrevivência) dos cultivares ao manejo; as diferenças morfofisiológicas entre os cultivares sob desfolhações e na condição final; e a existência de compensação entre os fatores de manejo (altura e intervalo) para a rebrota dos cultivares.

\section{Material e Métodos}

O trabalho foi conduzido em Passo Fundo no período de janeiro a agosto de 2005. Passo Fundo localiza-se na região do Planalto Médio, ao norte do Rio Grande do Sul, a uma latitude de $28^{\circ} 15^{\prime} \mathrm{S}, 52^{\circ} 24^{\prime} \mathrm{W}$ e $687 \mathrm{~m}$ de altitude. O clima é temperado subtropical úmido (Cfa), com temperatura média anual de $22^{\circ} \mathrm{C}$ (Moreno, 1961). Na Figura 1 constam os dados de temperatura média mensal durante o período experimental e as normais regionais. O ensaio constou da avaliação de três cultivares de Lotus spp., dois de cornichão (São Gabriel, ARS2620) e um de cornichão-dosbanhados (Maku), manejados sob regimes de corte e combinação de duas alturas (4 - $8 \mathrm{~cm}$ ) e dois intervalos de corte

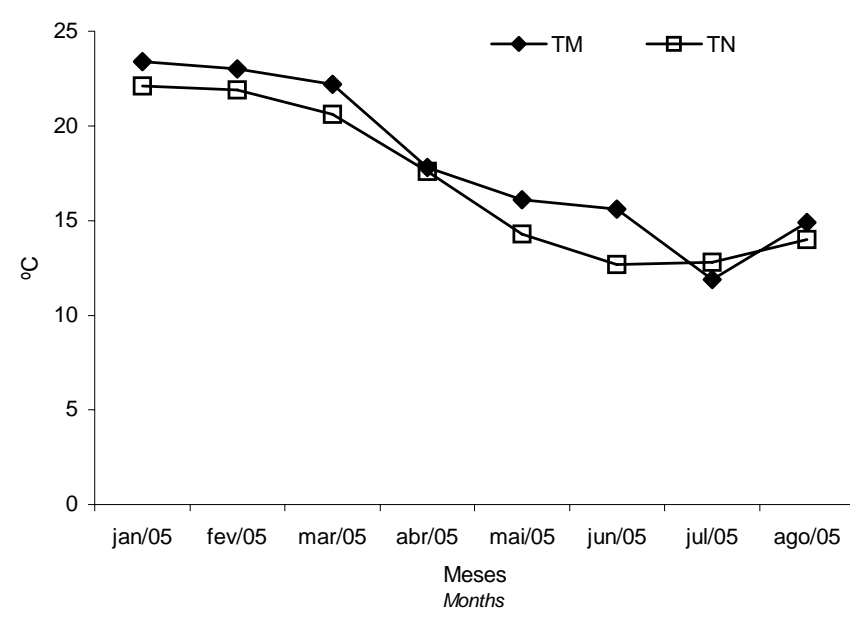

Figura 1 - Temperaturas médias mensais do período experimental (TM) e normais regionais (TN). Passo Fundo, RS. Fonte: www.cnpt.embrapa.br.

Figure 1 - Average monthly temperature during the experimental period (TM ) and regional normal (TN). Passo Fundo, RS. Fonte: www.cnpt.embrapa.br. 
(quinzenal - mensal), em esquema trifatorial. O delineamento experimental foi em blocos casualizados, com cinco repetições.

O cultivo foi realizado em ambiente semiprotegido, constituído de um telado com cobertura impermeável transparente e com proteção lateral de tela plástica do tipo clarite, sem cortinas. As plantas foram cultivadas em vasos plásticos com capacidade para $5 \mathrm{~kg}$ de solo seco ao ar, que constituíram as unidades experimentais. O período experimental ocorreu entre janeiro e agosto de 2005, totalizando 233 dias. As plântulas foram obtidas por meio de semeadura (7/1/2005) em bandejas multicelulares de isopor e mantidas em telado com irrigação automática com microaspersores. Em 18 de fevereiro de 2005, procedeu-se ao transplante para os vasos, preenchidos com substrato composto de solo proveniente de área de lavoura, camas sobrepostas de suíno à base de maravalha e casca de arroz e areia, na proporção de 75:15:10. A análise química desse substrato comprovou as seguintes características: $\mathrm{pH}$ : 5,4\%; P: $51 \mathrm{mg} / \mathrm{L}$; K: $195 \mathrm{mg} / \mathrm{L}$; argila: 26\%; MO: 4,3\%; Al: $0,0 \mathrm{cmol}_{\mathrm{C}} / \mathrm{dm}^{3}$, Ca: 4,3 $\mathrm{cmol}_{\mathrm{c}} / \mathrm{dm}^{3} ; \mathrm{Mg}: 3,2 \mathrm{cmol}_{\mathrm{c}} / \mathrm{dm}^{3}$; $\mathrm{H}+\mathrm{Al}: 3,5 \mathrm{cmol}_{\mathrm{c}} / \mathrm{dm}^{3}$ e CTC: $11,4 \mathrm{cmol}_{\mathrm{C}} / \mathrm{dm}^{3}$. As características do substrato estavam de acordo com as exigências da cultura das espécies em estudo e, portanto, não houve necessidade de fertilização. Aos 30 dias do transplante, foi realizada a inoculação mediante irrigação com solução de Rhizobium lotii a $2 \mathrm{~g} / \mathrm{L}$. Aos 60 dias da semeadura, foram aplicados $250 \mathrm{~mL} /$ vaso de uma solução de uréia a 3\%. Como tratamento inseticida, foram feitas duas aplicações de Azodrin 400, na dose de $1 \mathrm{~mL} / \mathrm{L}$, por meio de pulverização.

Em 12 de abril, as plantas foram submetidas ao primeiro corte e, posteriormente, foram cortadas de acordo com os intervalos pré-determinados: a) cortes quinzenais: 26/4/2005, 10/5/2005, 24/5/2005, 7/6/2005, 21/6/2005, 5/7/2005, 19/7/2005, 2/8/2005, 16/8/2005 e 30/8/2005; eb) cortes mensais: 10/5/2005, 7/6/2005, 5/7/2005, 2/8/2005 e $30 / 8 / 2005$. Os cortes foram realizados com tesoura de jardinagem. Em cada colheita, foram medidos a estatura e o diâmetro das plantas, respeitando seu caimento natural nos vasos. Depois de cada corte, foram atribuídas notas para a área foliar residual, segundo o critério: 1 = baixa; 3 = média; 5 = alta; para isso, utilizou-se um arco plástico colocado sobre a área residual das plantas, onde era feita a leitura. O material vegetal colhido foi levado ao laboratório para contagem das hastes, determinação do comprimento da maior haste e separação de hastes e folhas. As folhas e hastes foram secas em estufa de ventilação forçada a $60^{\circ} \mathrm{C}$ por 72 horas e, então, foram pesadas. Aos 233 dias da semeadura, procedeu-se à colheita final, ocasião em que as plantas foram retiradas dos vasos, lavadas e avaliadas, incluindo o sistema subterrâneo. Nessa colheita, além das variáveis avaliadas no decorrer dos demais cortes, foram medidos o diâmetro e comprimento da coroa e a extensão da massa radical (comprimento entre a base e o ápice do sistema radical). As raízes foram secas em estufa, como adotado para os demais componentes citados anteriormente. Os resultados foram submetidos à análise de variância e as médias foram comparadas pelo teste Tukey a $1 \%$ de significância.

\section{Resultados e Discussão}

O estabelecimento das plantas de Lotus spp. foi lento, como relatado por outros pesquisadores (Hughes, 1981; Paim \& Riboldi, 1991; Scheffer-Basso et al., 2001). Somente foi possível iniciar os cortes aos 95 dias após a semeadura. Nessa idade, os cultivares diferiram quanto à estatura, ao diâmetro, ao comprimento da maior haste, ao número de hastes e ao teor de matéria seca (MS) (Tabela 1).

Os cultivares S. Gabriel e Maku apresentaram hábito mais ereto e maior estatura em relação ao cultivar ARS2620, de hábito mais prostrado. A busca por plantas forrageiras de menor estatura, especialmente leguminosas, normalmente está vinculada à maior proteção de sítios meristemáticos. Briske (1996) aponta plantas de baixa estatura como tolerantes ao pastejo, pois apresentam mecanismo de escape por terem protegido na base grande número de folhas residuais e gemas basilares.

Apesar da pequena estatura, a produção de hastes do cultivar ARS2620 foi maior em relação à do cultivar São

Tabela 1 - Caracteres morfofisiológicos de cultivares de Lotus spp. ao primeiro corte, 95 dias após a semeadura

Table 1 - Morphophysiological characteristics of Lotus spp. cultivars in the first cutting time, 95 days after the seeding

\begin{tabular}{|c|c|c|c|}
\hline \multirow{2}{*}{$\begin{array}{l}\text { Característica } \\
\text { morfofisiológica } \\
\text { Morphophysiological } \\
\text { characteristic }\end{array}$} & \multicolumn{3}{|c|}{ Cultivar } \\
\hline & S. Gabriel & ARS2620 & Maku \\
\hline $\begin{array}{l}\text { Estatura }(\mathrm{cm}) \\
\text { Height }\end{array}$ & $17,4 \mathrm{~A}$ & $9,4 \mathrm{~B}$ & $16,4 \mathrm{~A}$ \\
\hline $\begin{array}{l}\text { Diâmetro da planta }(\mathrm{cm}) \\
\text { Plant diameter }\end{array}$ & $81,8 \mathrm{~A}$ & $71,3 \mathrm{~B}$ & $71,0 \mathrm{~B}$ \\
\hline $\begin{array}{l}\text { Comprimento da maior } \\
\text { haste }(\mathrm{cm})\end{array}$ & $22,0 \mathrm{~A}$ & $20,1 \mathrm{~B}$ & $21,7 \mathrm{~A}$ \\
\hline $\begin{array}{l}\text { Length of the largest stem } \\
\text { Hastes ( } \mathrm{n} \text {-/planta) }\end{array}$ & $26,7 \mathrm{C}$ & $41,2 \mathrm{~B}$ & $52,3 \mathrm{~A}$ \\
\hline $\begin{array}{l}\text { Stems (n./plant) } \\
\text { Massa seca (g/planta) } \\
\text { Dry matter (g/plant) }\end{array}$ & 1,7B & $1,8 \mathrm{~B}$ & $2,7 \mathrm{~A}$ \\
\hline
\end{tabular}

Médias seguidas de mesma letra não diferem $(P>0,01)$ pelo teste Tukey. Means followed by the same letter do not differ $(P>0.01)$ by Tukey test. 
Gabriel, evidenciando um processo de ramificação intenso nessa fase inicial, altamente interessante para o estabelecimento e a posterior rebrota. Essa expressiva ramificação compensou a baixa estatura e resultou em uma produção de MS similar à do cultivar São Gabriel. A maior quantidade de hastes e de MS foi obtida com cv. Maku, assim como estimado por Scheffer-Basso et al. (2002) nesse cultivar em comparação ao S. Gabriel, o que sugere maior precocidade ou estabelecimento mais rápido. O cultivar São Gabriel superou os demais quanto ao diâmetro das plantas, o que está de acordo com os dados descritos por Soster et al. (2004b), que obtiveram em campo $77 \mathrm{~cm}$ nesse cultivar em comparação ao ARS2620 (43 cm) aos 150 dias de crescimento.

O manejo de cortes afetou significativamente a produção de MS total no período avaliado, indicando interação de altura e intervalo com cultivar (Figura 2). Na combinação da maior freqüência (cortes quinzenais) e da menor altura (4 cm) de corte, os cultivares não evidenciaram diferenças, o que indica elevado dano às plantas, que não apresentaram mecanismos distintos de rebrota. No entanto, sob desfolhações mais leves, com maior intervalo e altura, as diferenças se manifestaram. Nesta condição, os cultivares São Gabriel e Maku foram superiores ao ARS2620, o que está relacionado à sua menor dimensão. Soster et al. (2004b) também obtiveram menor produção no cultivar ARS2620 em relação ao São Gabriel, no campo. Com as combinações intermediárias de altura $\times$ intervalo de corte (quinzenal $-8 \mathrm{~cm}$; mensal - $4 \mathrm{~cm}$ ), a resposta de cada cultivar foi a mesma e, em ambos os

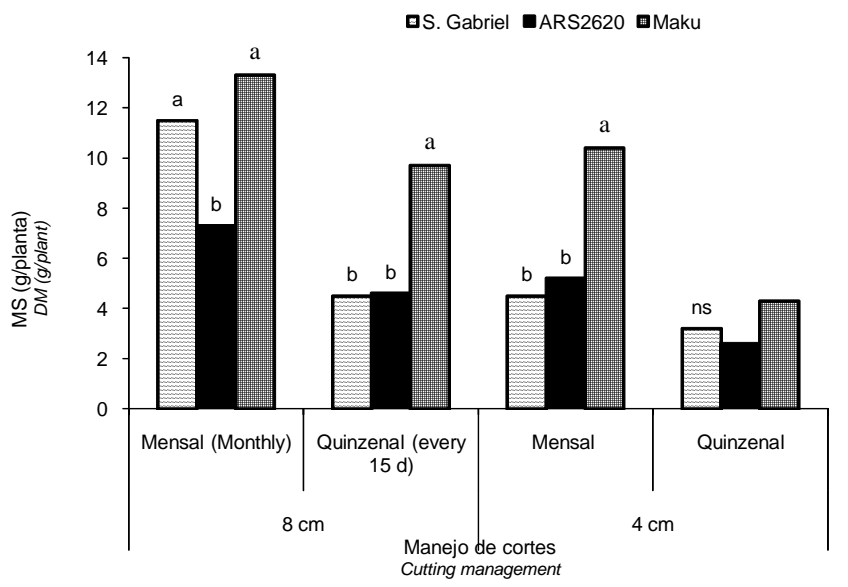

Figura 2 - Efeito da combinação de intervalo e altura de corte sobre a produção total de MS aérea de cultivares de Lotus spp.

Letras distintas sobre as colunas, dentro de cada combinação de fatores, indicam diferença significativa $(P<0,01)$ entre cultivares.

Figure 2 - Effect of the combination of cutting interval and cutting height on the total production of shoot dry DM of Lotus spp. cultivars.

Different letters in a column, within each factor combination, show significant difference $(P<0.01)$ among cultivars. casos, o cultivar Maku superou os demais. Neste caso, o cultivar São Gabriel teve sua produção reduzida e mostrou-se mais sensível que o Maku a regimes mais intensos de desfolhação. A resposta dos cultivares nos dois casos evidenciou um mecanismo compensatório e de plasticidade das plantas, de modo que, sob menor altura residual e maior intervalo, em situação oposta foi o mesmo. Araújo \& Jacques (1974a) ressaltaram a importância de se considerar os caracteres morfológicos do cornichão no estabelecimento de práticas de manejo visando maior produção de MS. Para o cultivar São Gabriel, esses autores obtiveram maior produção e vigor com cortes a $6 \mathrm{~cm}$ em relação a $3 \mathrm{~cm}$, em virtude da maior preservação das gemas axilares, principais responsáveis pela sua rebrota do cornichão.

Segundo Nelson \& Moser (1994), leguminosas mais prostradas geralmente apresentam menor produção em relação às de hábito mais ereto, apesar da vantagem de reterem maior área foliar próximo à superfície do solo quando pastejadas. Neste trabalho, a maior estatura dos cultivares foi obtida com o corte em menor freqüência e intensidade (Figura 3).

Segundo Greub \& Wedin (1971), o cornichão é beneficiado em intervalos de corte menos freqüentes; no entanto, é necessário deixar altura residual maior para manutenção da produção e persistência. Para o cultivar Maku, que tolera cortes mais intensos e em intervalos mais curtos, são exigidas longas rotações para manter sua persistência e competitividade e servir de estratégia para manter o equilíbrio entre a produção de rizomas e a produção de forragem (Harris et al., 1997; Sheat, 1980).

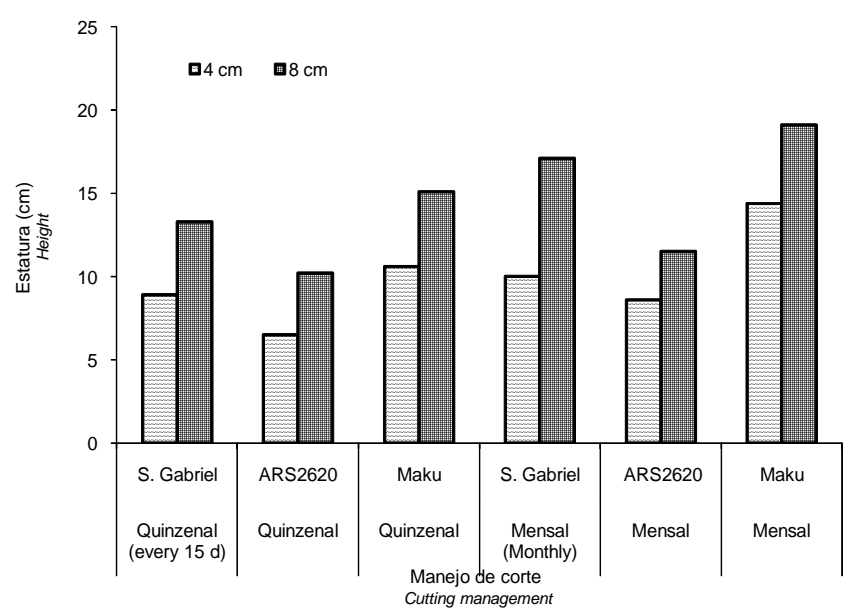

Figura 3 - Efeito da altura de corte na estatura média de cultivares de Lotus spp. manejados em diferentes freqüências de corte.

Figure 3 - Effect of the cutting height on the average height of Lotus spp. cultivars under different defoliation managements. 
Quanto à sobrevivência das plantas, somente em cortes mais drásticos (quinzenal - $4 \mathrm{~cm}$ ) houve morte de plantas, e apenas no cultivar São Gabriel (dados não apresentados). Esse cultivar teve 60\% de sobrevivência em relação a 100\% das demais, indicando maior vulnerabilidade às desfolhações mais intensas e freqüentes, atribuída à maior exposição de gemas axilares, em razão de seu hábito mais ereto em comparação a outros cultivares. Soster et al. (2004b), sob cortes mensais a $8 \mathrm{~cm}$ de altura, no campo, obtiveram 80 a 100\% de sobrevivência em populações de cornichão. Sob cortes, Duell \& Gausman (1957) também obtiveram redução do estande de cornichão quando o submeteram a cortes baixos (2,5 cm). De acordo com Araújo \& Jacques (1974b), cortes baixos em plantas jovens ocasionam esgotamento mais rápido das reservas orgânicas, o que comprova a importância de se manter o equilíbrio entre altura e intervalo de corte objetivando maior produção e, principalmente, aumentando o tempo de vida das plantas, dandolhes tempo maior para reporem suas reservas e persistirem sob pastejo.

A cobertura foliar residual foi afetada pelo manejo de corte e reduziu na combinação de cortes quinzenais a $4 \mathrm{~cm}$. O cultivar ARS2620 foi o menos afetado pelos cortes e, durante o período avaliado, apresentou sempre resíduo de médio a alto (Figura 4) e melhor recuperação relativa. Plantas forrageiras com essa característica podem ser classificadas como resistentes ao pastejo por mecanismo de escape (Briske, 1996). Esse resultado está relacionado à menor estatura do cultivar ARS2620 e à constante emissão de folhas e hastes pelo fato de as gemas estarem em nível basilar.

Segundo Brummer \& Bouton (1992), plantas com maior área foliar residual possuem maior fonte de fotoassimilados e não dependem somente da raiz para se regenerarem. O cultivar São Gabriel foi o mais afetado pelos cortes, o que confirma relatos de Greub \& Wedin (1971) e Araújo \& Jacques (1974a) de que cortes baixos em cornichão causam maior remoção das gemas e folhas axilares. No entanto, à medida que o intervalo de corte aumentou, mesmo na altura de $4 \mathrm{~cm}$, o resíduo foliar também aumentou; com isso, a capacidade de rebrota das plantas também melhorou, caracterizando a importância de determinado tempo entre pastejos para que o cornichão rebrote. O cultivar Maku se assemelhou mais ao S. Gabriel que ao ARS2620 e respondeu no mesmo nível, porém com maiores chances de persistência por ter se mantido com resíduo foliar médio a alto. De acordo com Duell \& Gausman (1957), mesmo que a planta apresente boa resposta a cortes baixos, a longevidade da pastagem fica comprometida.
Ao término do período de cultivo, aos 233 dias após a semeadura, os cultivares evidenciaram condições distintas relativas a medidas lineares e de peso (Tabela 2).

O cultivar Maku apresentou o maior $(\mathrm{P}<0,01)$ diâmetro de planta e de coroa, o que sugere maior vigor. No entanto, apresentou o menor comprimento de sistema radical, confirmando relatos de Lowther (1980) e Wendderburn \& Gwynne (1981), que caracterizaram o sistema radical desse cultivar como superficial, com raízes curtas e rizomas densamente enraizados, características de plantas adaptadas a solos pobres em fósforo e com alto teor de umidade. Quanto às alturas de corte, em todas as variáveis analisadas, obtiveram-se melhores respostas no maior nível residual (8 cm) (Tabela 3). Os resultados estão de acordo com os obtidos por Araújo \& Jacques (1974a), que observaram na avaliação residual efeitos benéficos dos cortes a $6 \mathrm{~cm}$ em relação aos de $3 \mathrm{~cm}$.

O sistema subterrâneo mostrou alterações significativas quanto à produção de MS e ao volume de raízes, que aumentaram em 120 e $157 \%$ quando a intensidade de corte foi reduzida de $4 \mathrm{~cm}$ para $8 \mathrm{~cm}$. Além disso, nesse sistema de manejo, a produção de hastes foi $141 \%$ maior ao corte mais drástico, disponibilizando mais pontos de crescimento. Em estudo com o cultivar Maku, Harris et al. (1997) ressaltaram que sob manejo intenso, na combinação de cortes a $2 \mathrm{~cm}$ e a cada 4 semanas, houve redução severa no desen-

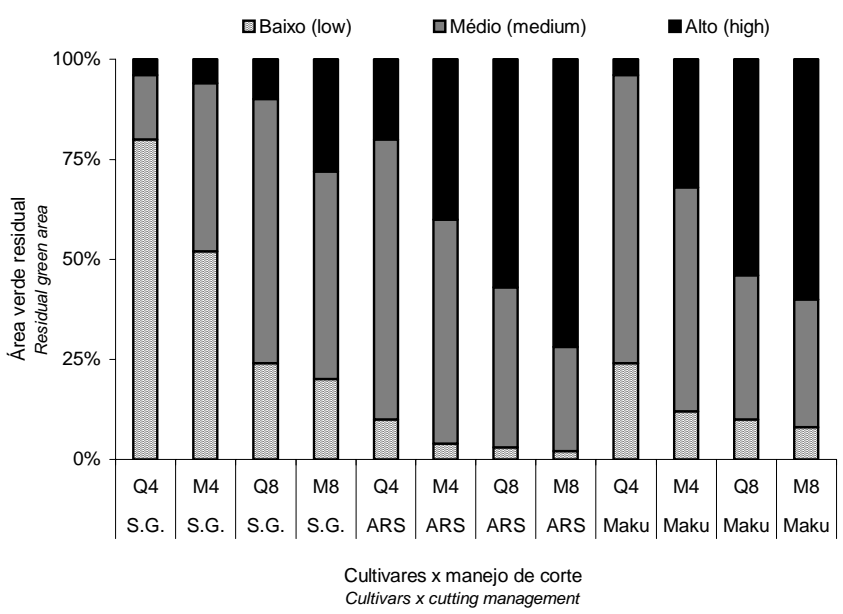

Figura 4 - Área verde residual de cultivares de Lotus spp. (S.G. = S. Gabriel; ARS = ARS2620; Maku) sob diferentes manejos de corte $(\mathrm{Q}=$ quinzenal, $\mathrm{M}=$ mensal; altura: 4 - $8 \mathrm{~cm}$ ), estimada em cada época de corte $[1=$ baixa $3=$ média; 5 = alta].

Figure 4 - Residual green area of Lotus spp. cultivars (S.G. = S. Gabriel; ARS= ARS2620; Maku) under different cutting management ( $Q=$ every $15 \mathrm{~d}$; $M=$ =monthly; height: 4 - $8 \mathrm{~cm}$ ), estimated in each cutting time $[1=$ low; $3=$ medium; $5=$ high) 
Tabela 2 - Caracteres morfofisológicos de Lotus spp. à última colheita

Table 2 - Morphophysiological characters of Lotus spp. cultivars at the last harvest

\begin{tabular}{llll}
\hline Característica morfofisiológica & \multicolumn{3}{c}{ Cultivar* } \\
\cline { 2 - 4 } Morphophysiological character & & \\
& S. Gabriel & ARS2620 & Maku
\end{tabular}

Dimensão

Size

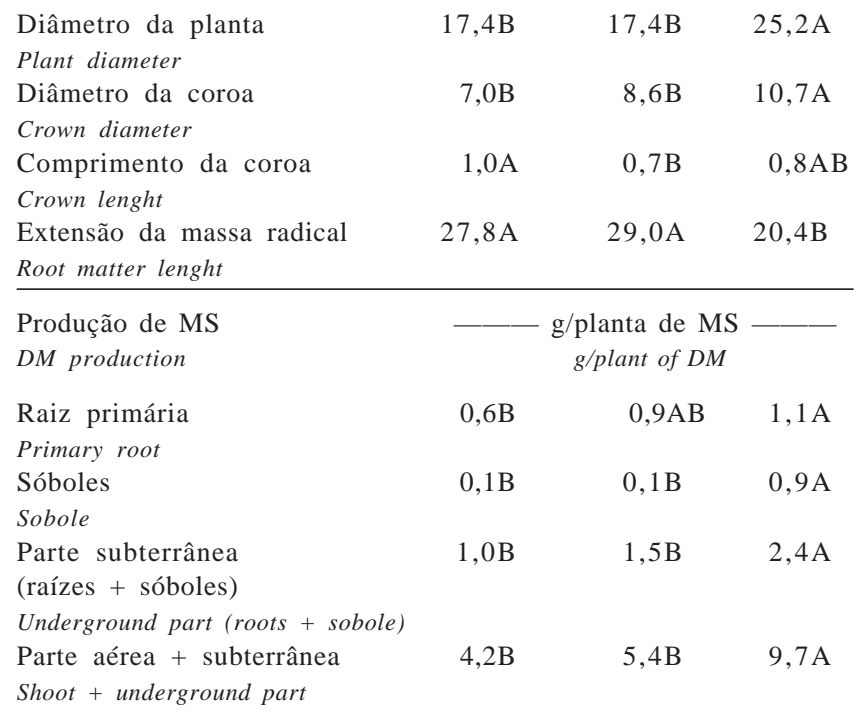

Médias seguidas de mesma letra na linha não diferem pelo teste Tukey $(\mathrm{P}>0.01)$.

* Na média do manejo de corte.

Means followed by the same letter in the row do not differ $(P>0.01)$ by Tukey test. *Average of cutting management.

volvimento subterrâneo, principalmente na produção dos sóboles; no entanto, mesmo a $2 \mathrm{~cm}$, mas com intervalo de oito semanas, a produção desses caules aumentou significativamente, o que indica a importância de uma compensação entre os fatores de manejo. Caso haja maior área foliar após o corte, possivelmente existirá menor gasto de reservas e também recuperação mais rápida da planta, de modo que a planta passa a armazenar reservas mais cedo, aumentando a quantidade total de glicídios disponíveis nas raízes e na base dos caules (Araújo \& Jacques, 1974b).

Os cultivares apresentaram respostas distintas às alturas de corte quanto à produção de hastes e parte aérea (Tabela 4). O cultivar que apresentou a maior produção residual de MS de hastes e parte aérea foi o Maku, caracterizando seu maior potencial de rebrota, atribuído ao seu hábito subterrâneo. Apesar do sistema radical pouco profundo, esse cultivar produz grande quantidade de sóboles, que emergem do solo e dão origem a novas hastes aéreas, o que, somando-se às hastes da coroa, permite maior resíduo de hastes após a desfolhação. A maior tolerância ao pastejo por espécies com essas características foi relatada por Nelson \& Moser (1994), Briske (1996) e Soster et al.
Tabela 3 - Efeito da altura de corte sobre características morfofisiológicas de cultivares de Lotus spp. na colheita final

Table 3 - Effect of cutting height upon morphophysiological characters of Lotus spp. cultivars at the last harvest

\begin{tabular}{|c|c|c|}
\hline \multirow[t]{2}{*}{$\begin{array}{l}\text { Característica morfofisiológica } \\
\text { Morphophysiological character }\end{array}$} & \multicolumn{2}{|c|}{$\begin{array}{l}\text { Altura de corte } \\
\text { Cutting height }\end{array}$} \\
\hline & $4 \mathrm{~cm}$ & $8 \mathrm{~cm}$ \\
\hline $\begin{array}{l}\text { Dimensão } \\
\text { Size }\end{array}$ & \multicolumn{2}{|c|}{$\longrightarrow \mathrm{cm}$} \\
\hline $\begin{array}{l}\text { Diâmetro da planta } \\
\text { Plant diameter }\end{array}$ & $16,4 \mathrm{~B}$ & $23,5 \mathrm{~A}$ \\
\hline $\begin{array}{l}\text { Diâmetro da coroa } \\
\text { Crown diameter }\end{array}$ & $7,8 \mathrm{~B}$ & $9,8 \mathrm{~A}$ \\
\hline $\begin{array}{l}\text { Produção de MS } \\
\text { DM production }\end{array}$ & \multicolumn{2}{|c|}{$\begin{array}{r}\text { g/planta deMS }- \\
\text { g/plant of } D M\end{array}$} \\
\hline $\begin{array}{l}\text { Hastes } \\
\text { Stems }\end{array}$ & $2,2 \mathrm{~B}$ & $5,3 \mathrm{~A}$ \\
\hline $\begin{array}{l}\text { Raiz primária } \\
\text { Primary root }\end{array}$ & $0,6 \mathrm{~B}$ & $1,1 \mathrm{~A}$ \\
\hline $\begin{array}{l}\text { Raízes secundárias } \\
\text { Secondary roots }\end{array}$ & $0,2 \mathrm{~B}$ & $0,7 \mathrm{~A}$ \\
\hline $\begin{array}{l}\text { Parte subterrânea (raízes+sóboles) } \\
\text { Underground part (roots+sobole) part }\end{array}$ & $1,0 \mathrm{~B}$ & $2,2 \mathrm{~A}$ \\
\hline $\begin{array}{l}\text { Parte aérea }+ \text { subterrânea } \\
\text { Shoot }+ \text { underground part }\end{array}$ & $3,8 \mathrm{~B}$ & $9,0 \mathrm{~A}$ \\
\hline $\begin{array}{l}\text { Volume radical }\left(\mathrm{cm}^{3}\right) \\
\text { Root volume }\end{array}$ & $6,1 \mathrm{~B}$ & $15,7 \mathrm{~A}$ \\
\hline
\end{tabular}

(2004a). O efeito do intervalo de cortes na condição final das plantas evidenciou a importância de maior período de descanso para a recuperação das plantas (Tabela 5).

Somente a MS de raiz primária não foi afetada pela freqüência de cortes, no entanto, essa raiz teve pouca importância em relação às demais, secundárias e fibrosas, formadas por Lotus spp. Assim, no conjunto de raízes, o volume das raízes foi significativamente reduzido (-39\%) em cortes mais freqüentes, comprovando o efeito do manejo sobre o sistema subterrâneo, fundamental para a rebrota por ser o local de reservas, absorção e sítios de nodulação. Na parte aérea residual, obteve-se aumento de 43,4\% em intervalo de quatro semanas em relação ao de duas semanas. Os resultados obtidos neste estudo foram similares aos descritos por Ayala et al. (2002) em seus trabalhos, realizados na Nova Zelândia e no Uruguai, com cornichão. Esse autor verificou que desfolhação intensa $(2 \mathrm{~cm})$ na primavera reduziu a produção de MS (17\%) e a população de plantas (21\%) em comparação a desfolhação leve (6 e 10 cm). A massa radical e da coroa, assim como o número de hastes e de reservas orgânicas nas raízes, reduziu sob corte intenso. Os efeitos da desfolhação foram confirmados posteriormente pelo autor em casa de vegeração. 
Tabela 4 - Efeito da interação cultivar $\times$ altura de corte sobre a produção de MS de Lotus spp. na colheita final

Table 4 - Effect of cultivar $x$ cutting height interaction on DM production of Lotus spp. at the last harvest

\begin{tabular}{lcc}
\hline $\begin{array}{l}\text { Componente } \\
\text { Component }\end{array}$ & Cultivar & \multicolumn{2}{c}{$\begin{array}{c}\text { Altura de corte } \\
\text { Cutting height }\end{array}$} \\
\cline { 2 - 3 } & \multicolumn{2}{c}{$8 \mathrm{~cm}$} \\
\hline & \multicolumn{2}{c}{$g$ de MS/planta $-\mathrm{gM} /$ plant } \\
\hline
\end{tabular}

Hastes residuais após o corte

Residual stems after cutting

$\begin{array}{ccc}\text { S. Gabriel } & 1,1 \mathrm{bB} & 3,6 \mathrm{bA} \\ \text { ARS2620 } & 1,7 \mathrm{bB} & 4,0 \mathrm{bA} \\ \text { Maku } & 3,6 \mathrm{aB} & 8,3 \mathrm{aA}\end{array}$

Parte aérea residual após corte

Residual shoot after cutting

$\begin{array}{crr}\text { S. Gabriel } & 1,5 \mathrm{bB} & 4,4 \mathrm{bA} \\ \text { ARS2620 } & 2,2 \mathrm{bB} & 5,6 \mathrm{bA} \\ \text { Maku } & 4,3 \mathrm{aB} & 10,3 \mathrm{aA}\end{array}$

Médias seguidas de mesma letra maiúscula na linha e minúscula na coluna não diferem $(P>0,01)$ pelo teste Tukey.

Means followed by the same letter, capital letter in the row and small in the column, do not $\operatorname{differ}(P>0.01)$ by Tukey test.

Tabela 5 - Características morfofisiológicas de Lotus spp. na colheita final manejado em diferentes intervalos de corte

Table 5 - Effect of cutting interval upon morphophysiological characters of Lotus spp., at the last harvest

\begin{tabular}{|c|c|c|}
\hline \multirow[t]{2}{*}{$\begin{array}{l}\text { Característica morfofisiológica } \\
\text { Morphophysiological character }\end{array}$} & \multicolumn{2}{|c|}{$\begin{array}{l}\text { Freqüência de corte } \\
\text { Cutting height }\end{array}$} \\
\hline & $\begin{array}{l}\text { Semanal } \\
\text { Every } 15 d\end{array}$ & $\begin{array}{l}\text { Mensal } \\
\text { Monthly }\end{array}$ \\
\hline $\begin{array}{l}\text { Diâmetro da planta }(\mathrm{cm}) \\
\text { Plant diameter }\end{array}$ & $19,2 \mathrm{~B}$ & $20,9 \mathrm{~A}$ \\
\hline $\begin{array}{l}\text { Produção de massa seca } \\
\text { Dry matter production }\end{array}$ & - $\mathrm{g}$ de & $\mathrm{a}$ \\
\hline $\begin{array}{l}\text { Raiz primária } \\
\text { Primary root }\end{array}$ & $0,7 \mathrm{~A}$ & $0,9 \mathrm{~A}$ \\
\hline $\begin{array}{l}\text { Hastes } \\
\text { Stems }\end{array}$ & $3,0 \mathrm{~B}$ & $4,6 \mathrm{~A}$ \\
\hline $\begin{array}{l}\text { Parte aérea } \\
\text { Shoot }\end{array}$ & $4,0 \mathrm{~B}$ & $5,7 \mathrm{~A}$ \\
\hline $\begin{array}{l}\text { Parte subterrânea } \\
\text { (raízes + sóboles) }\end{array}$ & $1,4 \mathrm{~B}$ & $2,0 \mathrm{~A}$ \\
\hline $\begin{array}{l}\text { Underground part (root }+ \text { sobole) } \\
\text { Parte aérea e subterrânea }\end{array}$ & $5,3 \mathrm{~B}$ & 7,6A \\
\hline $\begin{array}{l}\text { Shoot and underground part } \\
\text { Volume radical }\left(\mathrm{cm}^{3}\right) \\
\text { Root volume }\end{array}$ & $8,3 \mathrm{~B}$ & $13,7 \mathrm{~A}$ \\
\hline
\end{tabular}

Médias seguidas de mesma letra na linha não diferem $(P>0,01)$ pelo teste Tukey.

Means followed by the same letter in the row do not differ $(P>0.01)$ by Tukey test.

* Média dos cultivares (Cultivar average).

\section{Conclusões}

Os cultivares S. Gabriel e Maku superam o cultivar ARS2620 em produção de massa seca. O cultivar ARS2620 tem menor estatura e mantém maior cobertura foliar residual após corte, o que indica mecanismo de resistência ao pastejo do tipo escape.

Na condição residual pós-desfolhação, o cultivar Maku supera o S. Gabriel e o ARS2620 em diâmetro da coroa, massa seca de raiz primária, sóboles e produções subterrânea e aérea mais vigorosas, o que sugere maior persistência sob pastejo intenso.

Os cultivares de Lotus spp. são favorecidos em condições de manejo menos intenso e possuem mecanismo compensatório quando submetidos a cortes mais baixos, desde que sob menor freqüência, ou a cortes freqüente, desde que menos intensos. A persistência do cultivar São Gabriel é severamente afetada por cortes intensos e freqüentes.

\section{Literatura Citada}

APPEZZATO DA GLÓRIA, B. Morfologia de sistemas subterrâneos: história e evolução do conhecimento no Brasil. 1.ed. Ribeirão Preto: Editora e Arte, 2003. 80p.

ARAÚJO, J.C.; JACQUES, A.V.A. Características morfológicas e produção de matéria seca de cornichão (Lotus corniculatus L.) colhido em diferentes estádios de crescimento e a duas alturas corte. Revista Brasileira de Zootecnia, v.3, n.2, p.138-147, 1974a.

ARAÚJO, J.C.; JACQUES, A.V.A. Influência do estádio de crescimento e da altura de corte sobre as reservas de glicídios e nitrogênio total de cornichão (Lotus corniculatus L.). Revista Brasileira de Zootecnia, v.3, n.2, p.123-137, 1974b.

AYALA, W.; HODGSON, J.; KEMP, P.D. Effects of defoliation intensity on growth, biomass distribution, and morphological and physiological changes birdsfoot trefoil (Lotus corniculatus L.) in glasshouse conditions. Agronomy New Zealand, v.32, p.83-88, 2002.

BEUSELINCK, P.R.; STEINER, J.J. Registration of 'ARS-2620' birdsfoot tTrefoil. Crop Science, v.36, p.1414, 1996.

BRISKE, D.D. Strategies of plant survival in grazed systems a functional interpretation. In: HODGSON, J.; ILLIUS, W. (Eds). The ecology and management of grazed systems. Wallingford: CAB, 1996. p.37-67.

BROUGHAM, R.W. Effect of intensity of defoliation on regrowth of pasture. Australian Journal Agricultural Research, v.7, n.5, p.377-387, 1956.

BROWN, R.H.; COOPER, R.B.; BLASER, R.E. Effects of leaf age on efficiency. Crop Science, v.6, p.206-209, 1966.

BRUMMER, E.C.; BOUTON, J.H. Physiological traits associated with grazing-tolerance alfalfa. Agronomy Journal, v.84, n.2, p.138-143, 1992.

CAROSO, G.F.; PAIM, N.R.; PRATES, E.R. Avaliação da produção e persistência de progênies e cultivares de Lotus corniculatus L. Pesquisa Agropecuária Brasileira, v.16, n.3, p.341-346, 1981.

DUELL, R.W.; GAUSMAN, H.W. The effect of differential cutting on the yield, persistence, protein and mineral content of birdsfoot trefoil. Agronomy Journal, v.49, p.318-319, 1957. 
FLARESSO, J.A.; SAIBRO, J.C. Influência de regimes de cortes e adubação no rendimento de matéria seca, reservas de glicídios não-estruturais e ressemeadura natural de Lotus corniculatus L. Pesquisa Agropecuária Brasileira, v.27, n.1, p.181-188, 1992.

GREUB, L.F.; WEDIN, W.F. Leaf area, dry-matter accumulation and carbohydrate reserves of alfalfa and birdsfoot trefoil under a three-cut management. Crop Science, v.11, n.3, p.341-344, 1971.

HARRIS, C.A.; BLUMENTHAL, M.J.; KELMAN, W.M. et al. Effect of cutting height and cutting interval on rhizome development, herbage production and herbage quality of Lotus pedunculatus cv. Grasslands Maku. Australian Journal of Experimental Agriculture, v.37, p.631-637, 1997.

HUGHES, H.D. Cuernecillo. In: HUGHES, H.D.; HEATH, M.; METCALFE, D.S. (Eds.) Forages. Ciudad de Mexico: Compañia Editora Continental, 1981. p.215-232.

LOWTHER W.L. Establishment and growth of clovers and Lotus on acid soil. New Zealand Journal Experimental Agriculture, v.8, p.31-138, 1980.

MAROSO, R.P; CARNEIRO, C.M.; BORDIGNON, M.V. et al. Variabilidade morfológica do sistema subterrâneo de Lotus corniculatus L. Agrociencia, v.8, n.2, p.73-78. 2004.

MOREnO, J.A. Clima do Rio Grande do Sul. Porto Alegre: Secretaria da Agricultura, 1961. 41p.

NELSON, C.J.; MOSER, L.E. Plant factors affecting forage quality, In: FAHEY, J.C. (Ed.). Forage quality, evaluation, and utilization. Madison: American Society of Agronomy, 1994. p.115-154.

PAIM, N.R.; RIBOLDI, J. Comparação entre espécies e cultivares do gênero Lotus. Pesquisa Agropecuária Brasileira, v.26, p.1699-1701, 1991.

SCHEFFER-BASSO, S.M.; VOSS, M.; JACQUES, A.V.A. Nodulação e fixação biológica de nitrogênio de Adesmia latifolia e Lotus corniculatus em vasos de Leonard. Revista Brasileira de Zootecnia, v.30, n.3, p.687-693, 2001.
SCHEFFER-BASSO, S.M.; JACQUES, A.V.M.; DALL’AGNOL, M. Alocação da biomassa e correlações morfofisiológicas em leguminosas forrageiras com hábitos de crescimento contrastantes. Scientia Agricola, v.59, n.4, p.629-634, 2002.

SHEAFFER, C.C.; MARTEN, G.C.; JORDAN, R.M. et al. Forage potencial of kura clover and birdsfoot trefoil when grazed by sheep. Agronomy Journal, v.84, n.1, p.176-180, 1992.

SHEAT, G.W. Production and regrowth characteristics of Lotus pedunculatus Cav. Cv. "Grasslands Maku”. New Zealand Journal of Agricultural Research, v.23, n.2, p.191200, 1980 .

SMITH, D. Carbohydrate root reserves in alfalfa, red clover, and birdsfoot trefoil under several management schedules. Crop Science, v.2, n.75, p.75-78, 1962.

SOSTER, M.T.B; SCHEFFER-BASSO, S.M.; DALL'AGNOL, M. Caracterização morfofisiológica de genótipos de cornichão (Lotus corniculatus L.). Revista Brasileira de Zootecnia, v.33, n.6, p.1654-1661, 2004a.

SOSTER, M.T.B.; SCHEFFER-BASSO, S.M.; DALL'AGNOL, M. et al. Caracterização agronômica de genótipos de cornichão (Lotus corniculatus, L.) Revista Brasileira de Zootecnia, v.33, n.6, p.1662-1671, 2004b.

WEDDERBURN, M.E.; GWYNNE, D.C. Seasonality of rhizome and shoot production and fixation in Lotus uliginosus under upland conditions in South-West Scotland. Annals of Botany, v.48, n.1, p.8-13, 1981.

WEN, L.; KALLENBACH, R.L.; WILLIAMS, J.E. et al. Performance of steers grazing rhizomatous and nonrhizomatous birdsfoot trefoil in pure stands and in tall fescue mixtures. Journal of Animal Science, v.80, n.7, p.1970-1976, 2002.

Recebido: 20/10/2006 Aprovado: $12 / 4 / 2007$ 